\title{
Beppo-SAX OBSERVATION OF THE COMA CLUSTER
}

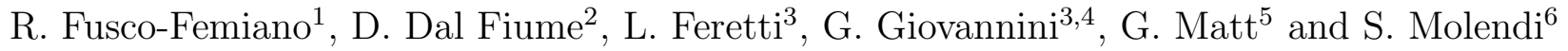 \\ ${ }^{1}$ Istituto di Astrofisica Spaziale, C.N.R., via del Fosso del Cavaliere, I-00133 Roma, Italy \\ 2 TESRE, C.N.R., via Gobetti 101, I-40129 Bologna, Italy \\ ${ }^{3}$ Istituto di Radioastronomia del CNR, Via P. Gobetti 101, I-40129 Bologna, Italy \\ ${ }^{4}$ Dipartimento di Fisica, Universitá di Bologna, via B. Pichat 6/2, 40126 Bologna, Italy \\ 5 Dip. di Fisica, Univ. "Roma Tre", via della Vasca Navale 84, I-00146 Roma, Italy \\ ${ }^{6}$ Istituto di Fisica Cosmica, C.N.R., Via Bassini 15, I-20133 Milano, Italy
}

\begin{abstract}
We present first results of the BeppoSAX observation of the Coma Cluster. Thanks to the unprecedented sensitivity of the PDS instrument, the source has been detected up to $\sim 80 \mathrm{keV}$. There is clear evidence for emission in excess to the thermal one above $\sim 25 \mathrm{keV}$, very likely of non-thermal origin. We have therefore, for the first time, detected the long sought Inverse Compton emission on CMB photons predicted in clusters, like Coma, with radio halos. Combining $\mathrm{X}$ and radio observations, a value of $0.16 \mu G$ for the volume-averaged intracluster magnetic field is derived.
\end{abstract}

\section{INTRODUCTION}

The Coma cluster has been observed in December 1997, as Beppo-SAX AO-1 observation, for an exposure time of $\sim 91 \mathrm{ksec}$ with the Medium Energy Concentrator Spectrometer (MECS) in the energy range 0.1-10 keV, and with the Phoswich Detection System (PDS) in the energy range 15-200 $\mathrm{keV}$. The main aim of this long observation was to search for non-thermal hard X-ray radiation (HXR) exploting the unique capabilities of the PDS: an overall sensitivity better than a few times $10^{-6} \mathrm{ph} / \mathrm{cm}^{2} \mathrm{~s} \mathrm{keV}$ in the energy band $40-80 \mathrm{keV}$, a small field of view (FWHM $=1^{\circ} .3$, hexagonal) in order to reduce source confusion and wide energy coverage to measure the tail of the thermal emission in the band 15-20 keV. Non-thermal hard X-ray radiation is predicted in galaxy clusters showing a radio halo, such as Coma $\mathrm{C}$ present in the central region of the Coma cluster (Willson 1970), due to Compton scattering of relativistic electrons by the Cosmic Microwave Background (CMB) photons. The expected value of the spectral index of the non-thermal HXR is $1+\alpha$, where $\alpha=1.34 \pm 0.06$ is the slope of the radio spectrum (Giovannini et al. 1993, ApJ, 406, 399). The combined radio and HXR detections would directly yield the opportunity to estimate the mean volume-averaged intracluster magnetic field, B, and the electron energy density, $\rho_{e}$. The most recent attempt to detect hard X-ray radiation in the spectrum of the Coma cluster is due to the OSSE experiment, onboard the Compton GRO. It reports upper limits that allow to get a lower limit on B of $0.1 \mu \mathrm{G}$ and an upper bound of $3.5 \times 10^{-13}$ ergs $\mathrm{cm}^{-3}$ on the energy density of electrons with energies $\geq 500 \mathrm{MeV}$ (Rephaeli, Ulmer \& Gruber 1994). To get information on $\rho_{e}$ it is necessary to know the size of the radio source and the distance of the cluster. The importance of these determinations is in that they are based essentially only on observables, whereas to determine $\mathrm{B}$ and $\rho_{e}$ from the radio data alone recourse must be made to additional theoretical assumptions, such as equipartion (Giovannini et al. 1993), or to polarization studies of the Faraday rotation (Kim et al. 1990; Feretti et al. 1995), but the results are model dependent. 


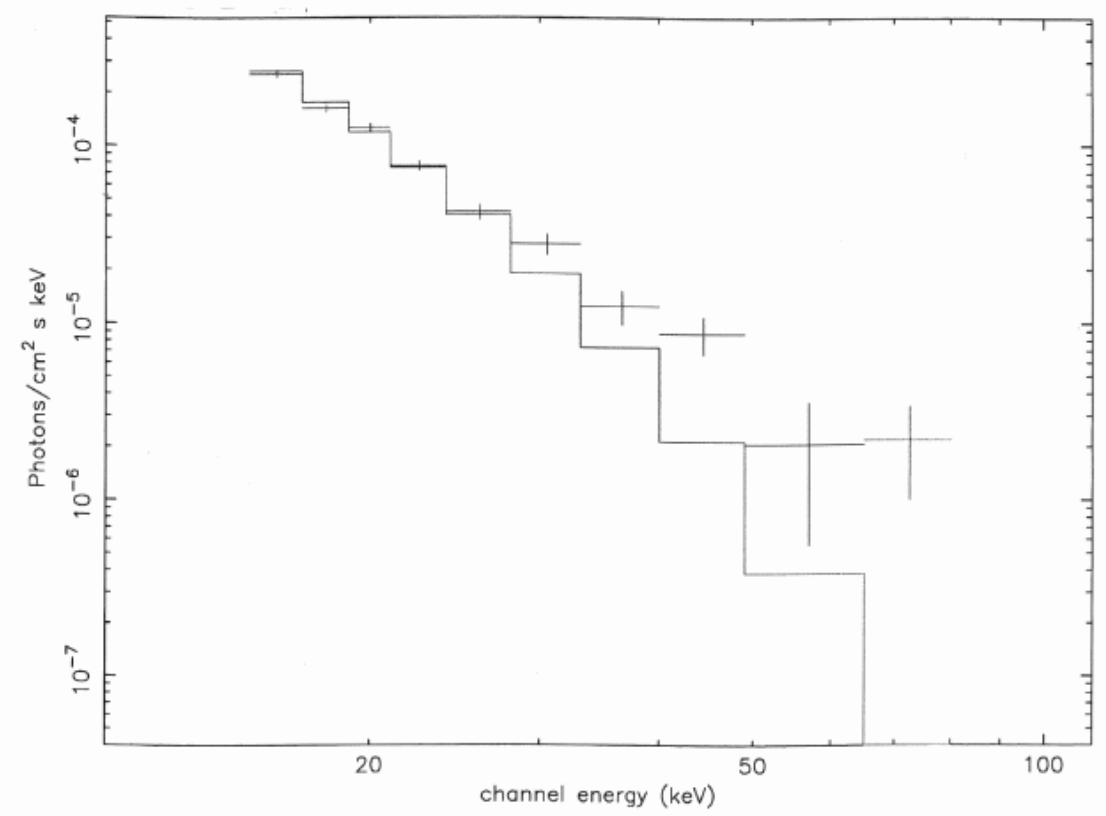

Figure 1: PDS data. The continuous line represents the best fit with a thermal component at the average cluster gas temperature of $8.21 \mathrm{keV}$ (Hughes et al. 1993). Error bars are $1 \sigma$

Throughout the paper we assume a Hubble constant of $H_{o}=50 \mathrm{~km} \mathrm{~s}^{-1} \mathrm{Mpc}^{-1}$. The angular distance of $1^{\prime}$ corresponds to $40.6 \mathrm{kpc}\left(z_{\text {Coma }}=0.0232\right)$.

\section{DATA ANALYSIS AND RESULTS}

The observation with the MECS allowed to perform spatially resolved spectroscopy in the core of the cluster with concentric annuli of $2^{\prime}$ within a region of $8^{\prime}$ in radius $(\sim 325 \mathrm{kpc})$. The data analysis does not show variations of the intracluster (IC) gas temperature and metallicity with the radius. The average temperature results to be $9.1 \pm 0.2 \mathrm{keV}$ and $0.257 \pm 0.026$ the average iron abundance. The temperature is somewhat larger than the typical value obtained by larger field-of-view, non imaging instruments (see below), confirming the presence of a temperature gradient. The $K_{\beta}$ over $K_{\alpha}$ line ratio is not well determined: $K_{\beta} / K_{\alpha}=0.09_{-0.09}^{+0.20}$; the expected value at $9 \mathrm{keV}$ being $\sim 0.17$ (Buote et al. 1998) for an optically thin plasma (quoted confidence intervals are at 90\%). The MECS results will be presented in detail in a forthcoming paper.

The PDS detected a hard X-ray emission in the energy range 15-80 keV in the Coma cluster spectrum, as shown in Fig. 1. The continuous line represents the best-fit with a thermal component at the average cluster gas temperature of $8.21( \pm 0.16) \mathrm{keV}$, as determined by the Large Area Counter (LAC) experiment onboard Ginga with a field of view of $1^{\circ} \times 2^{\circ}$ (Hughes et al. 1993), not much unlike that of the PDS $\left(1^{\circ} .3\right.$, hexagonal). Different temperatures, in the range $7.5-8.5 \mathrm{keV}$, have been reported by other X-ray observatories, but with a clear pattern: the larger the field of view, the lower the measured temperature. With the PDS data alone it is not possible to perform complex spectral fits, while MECS data are not of much help as its field of view is much smaller than that of the PDS. We therefore fixed the temperature of the thermal component to the accepted average cluster value of $8.21 \mathrm{keV}$. (But even adopting a temperature as high as $9.1 \mathrm{keV}$, as observed by the MECS 
in the cluster core, the results are substantially the same.) The flux of the thermal component is $\sim 3 \times 10^{-10} \mathrm{erg} \mathrm{cm} \mathrm{cm}^{-2} \mathrm{~s}^{-1}$ in the energy range $2-10 \mathrm{keV}$ (the Ginga flux is $3.4 \times 10^{-10} \mathrm{erg} \mathrm{cm}^{-2} \mathrm{~s}^{-1}$ in the same energy range); the fit, however, is very poor, with a reduced $\chi^{2}$ of 3.2 for 9 d.o.f., due to a clear excess above $25 \mathrm{keV}$ at a $4.5 \sigma$ level. The presence of a second component in the PDS spectrum may be deduced also by the fit with a single not fixed thermal component, which gives $\mathrm{kT}=10.71 \pm 0.76$ with a reduced $\chi^{2}=1.98$ for 8 d.o.f.: The $\chi^{2}$ value has a significant improvement by adding a second component, which may be due to : i) a non-thermal component, or ii) a second thermal component of temperature higher than $8.21 \mathrm{keV}$. Fitting the PDS data with a thermal component at the average cluster gas temperature of $8.21 \mathrm{keV}$ plus a non-thermal component, the reduced $\chi^{2}$ value is 0.92 for 7 d.o.f. with a large $1 \sigma$ confidence interval for the power law spectral index : 0.97-3.45; the flux of the non-thermal component is rather stable with respect to the variation of the spectral index $\left(\sim 2 \times 10^{-11} \mathrm{erg} \mathrm{cm}^{-2} \mathrm{~s}^{-1}\right.$ in the energy range 20-80 keV). As discussed in the Introduction, a non-thermal emission is predicted in clusters showing a radio halo, such as Coma $\mathrm{C}$, due to inverse Compton scattering of relativistic electrons by the $3 \mathrm{~K}$ microwave background photons. The theoretical value of the spectral index is 2.34. If we fix the spectral index at the theoretical value of 2.34, we obtain an excellent fit with a reduced $\chi^{2}$ of 0.83 for 8 d.o.f.; the flux of the thermal component $(\mathrm{kT}=8.21 \mathrm{keV})$ is $2.0 \times 10^{-11} \mathrm{erg} \mathrm{cm}^{-2} \mathrm{~s}^{-1}$ and $2.2 \times 10^{-11} \mathrm{erg} \mathrm{cm}^{-2} \mathrm{~s}^{-1}$ for the nonthermal emission. On the other hand, if we consider a second thermal component, instead of the non-thermal component, the fit requires a temperature greater than $40 \mathrm{keV}$. This unrealistic value may be interpreted as a strong indication that the detected hard excess is indeed due to a non-thermal mechanism.

It is of interest to remark that the X-ray fluxes measured by Ginga and PDS coincide, within the errors, in their common energy range $15-20 \mathrm{keV}$. The emission detected by the PDS and Ginga LAC is essentially the tail of the thermal radiation. Taking the theoretical value of 2.34 for the spectral index of the hard Compton flux, the contribution of this component is not greater than $20 \%$ at 20 $\mathrm{keV}$.

\section{DISCUSSION AND CONCLUSIONS}

The PDS onboard the Beppo-SAX satellite detected hard X-ray emission up to energies of $\sim 80 \mathrm{keV}$ in the spectrum of the Coma cluster. The first check was to examine the possibility that the observed hard emission is caused by contamination from a point source as an AGN. The sources present in the field of view of the PDS give a negligible contribution to the measured hard flux. The PDS data can be fitted by fixing one thermal component to the average cluster gas temperature of $\approx 8 \mathrm{keV}$, as determined by various X-ray experiments. The fluxes measured by the PDS and the Ginga LAC are in excellent agreement in their common energy range $15-20 \mathrm{keV}$. The field of view of the two experiments are approximately of the same order of size. In this energy range the PDS and Ginga LAC measure essentially the tail of the thermal component. As previously discussed, the fits to the PDS data show an evident excess of emission which is hardly explainable by a second thermal component, as the required temperature is unrealistically high $(>40 \mathrm{keV})$, thus favouring a non-thermal mechanism. The relativistic electrons, present in the IC medium of some clusters that show extended regions of radio emission, have Compton scattering with the $\mathrm{CMB}$ photons producing radiation that can reach $\mathrm{MeV}$ energies. Combining the syncrotron radio flux with the X-ray Compton flux it is possibile to derive, using only observables, the value of the mean volume-averaged $\mathrm{IC}$ magnetic field, $\mathrm{B}$, in the radio halo Coma $\mathrm{C}$ of size $\sim 1 \mathrm{Mpc}$, located in the central region of the Coma cluster. This determination is independent of the radio halo size and of the cluster distance. The radio data (Giovannini et al. 1993) and the $\mathrm{X}$-ray spectrum detected by the PDS implie a value of $\mathrm{B} \simeq 0.16 \mu G$. This value is not much 
different from the lower limit of $0.1 \mu G$ derived by the $2 \sigma$ upper bounds to the HXR reported by the OSSE experiment. At $\sim 50 \mathrm{keV}$, the HXR measurement of the PDS is lower by a factor $\sim 2$ with respect to the upper limit. Knowing the radius size, $\mathrm{R}(\simeq 1 \mathrm{Mpc})$, and the distance, $\mathrm{d}(\simeq 138 \mathrm{Mpc})$, of the radio source we estimate an energy density of $6.8 \times 10^{-14} \mathrm{ergs} / \mathrm{cm}^{3}$ for electrons with energies $\geq 500 \mathrm{MeV}$, using our derived value of B. Methods to determine the IC magnetic field strength, B, are based on the detection of energetic non-thermal photons, minimal arguments or measurements of Faraday rotation of polarized radiation observed through the ICM. We have already cited the lower limit of $0.1 \mu G$ by the OSSE experiment. A greater lower limit of $0.4 \mu G$ is derived from the upper limit to the gamma-ray radiation above $100 \mathrm{MeV}$, due to relativistic bremsstrahlung, obtained by the EGRET experiment (Sreekumar et al. 1996), assuming an extrapolation of the radio spectrum to lower frequencies. This model requires the presence of energetic cosmic rays in the cluster that interact with the hot ICM. Using minimal energy arguments, Giovannini et al. (1993) derive an equipartition magnetic field of $\sim 0.4 h_{50}^{2 / 7} \mu G$. Greater estimates of B are given by Faraday rotation measurements of the polarized radiation seen through the hot ICM of the Coma cluster, whose gas density profile is derived by X-ray observations. These values of B result $1.7 h_{50}^{1 / 2} \mu G$ (Kim et al. 1990) and $\sim 6 h_{50}^{1 / 2} \mu G$ (Feretti et al. 1995), although the last authors suggest also the presence of a weaker magnetic fiel component $\left(\sim 0.2 h_{50}^{1 / 2} \mu G\right)$, uniform on the cluster core radius scale. The value of the mean volume-averaged IC magnetic field, derived using the detection of non-thermal HXR by the $\operatorname{PDS}(\mathrm{B} \simeq 0.16 \mu G)$, is below the estimates resulting from the Faraday rotation measurements. This reflects the fact that the PDS observation measures the magnetic content of a large region of the Coma cluster. Besides, the value of $\mathrm{B}$ derived by the Faraday rotation measurements may be altered by the presence of local density peaks in a clumpy IC. Very attractive suggestions have been proposed regarding the origin of the IC magnetic field, as that in which galaxy motion may drive a turbulent dynamo in order to amplify faint seed fields to $\mu G$ fields. However, the origin of the diffuse IC magnetic field in clusters of galaxies has not yet been clarified. (Ruzmaikin et al. 1989; De Young 1992).

$\underline{\text { Acknowledgments }}$

We thank P.Grandi and L.Piro for useful suggestions regarding the data analysis.

\section{REFERENCES}

Buote, D.A., Canizares, C., Fabian ,A.C., and S.W. Allen 1998, astro-ph 9804290

De Young, D. 1992, ApJ, 386, 464

Feretti, L. Dallacasa, D., Giovannini, G., Tagliani, A. 1995, A\&, 302, 680

Giovannini, G., Feretti, L., Venturi, T, Kim, K.T., Kronberg, P.P. 1993, ApJ, 406, 399

Hughes, J.P., J.A. Butcher, G.C. Stewart, and Y. Tanaka 1993, ApJ, 404, 619

Kim, K.T., Kronberg, P.P., Dewdney, P.E., Landecker, T.L. 1990, ApJ, 355, 29

Rephaeli, Y., M. Ulmer, and D.E. Gruber 1994 ApJ, 429, 554

Ruzmaikin, A., Sokoloff, D. and Shukarov, A. 1989, MNRAS, 241, 1

Sreekumar et al. 1996, ApJ, 464, 628

Willson, M.A. 1970, MNRAS, 151, 1 